\title{
Class I histone deacetylases are major histone decrotonylases: evidence for critical and broad function of histone crotonylation in transcription
}

Wei Wei ${ }^{1,2, *}$, Xiaoguang Liu ${ }^{1, *}$, Jiwei Chen ${ }^{1, *}$, Shennan Gao ${ }^{1}, \mathrm{Lu} \mathrm{Lu}^{1}$, Huifang Zhang ${ }^{1}$, Guangjin Ding ${ }^{1}$, Zhiqiang Wang ${ }^{1}$, Zhongzhou Chen ${ }^{3}$, Tieliu Shi ${ }^{1}$, Jiwen $\mathrm{Li}^{1}$, Jianjun $\mathrm{Yu}^{4,5}$, Jiemin Wong ${ }^{1,2}$

${ }^{I}$ Shanghai Key Laboratory of Regulatory Biology, The Institute of Biomedical Sciences and School of Life Sciences, East China

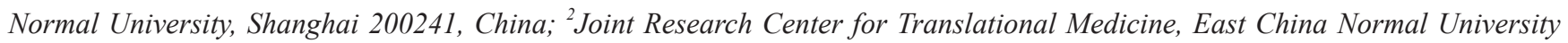
and Shanghai Fengxian District Central Hospital, No. 6600, Nanfeng Road, New Nanqiao Town, Fengxian District, Shanghai 201499, China, ${ }^{3}$ Beijing Advanced Innovation Center for Food Nutrition and Human Health, China Agricultural University, Beijing 100193, China; ${ }^{4}$ Department of Urology, Shanghai Jiao Tong University Affiliated Sixth People's Hospital, No. 600, Yishan Road, Xuhui District, Shanghai 200233, China; ${ }^{5}$ Department of Urology, Shanghai Sixth People's Hospital South Campus, No. 6600, Nanfeng Road, New Nanqiao Town, Fengxian District, Shanghai 201499, China

Recent studies on enzymes and reader proteins for histone crotonylation support a function of histone crotonylation in transcription. However, the enzyme(s) responsible for histone decrotonylation (HDCR) remains poorly defined. Moreover, it remains to be determined if histone crotonylation is physiologically significant and functionally distinct from or redundant to histone acetylation. Here we present evidence that class I histone deacetylases (HDACs) rather than sirtuin family deacetylases (SIRTs) are the major histone decrotonylases, and that histone crotonylation is as dynamic as histone acetylation in mammalian cells. Notably, we have generated novel HDAC1 and HDAC3 mutants with impaired HDAC but intact HDCR activity. Using these mutants we demonstrate that selective HDCR in mammalian cells correlates with a broad transcriptional repression and diminished promoter association of crotonylation but not acetylation reader proteins. Furthermore, we show that histone crotonylation is enriched in and required for self-renewal of mouse embryonic stem cells.

Keywords: class I histone deacetylases; histone decrotonylation; histone deacetylation; histone deacylation; HDAC; SIRT Cell Research (2017) 27:898-915. doi:10.1038/cr.2017.68; published online 12 May 2017

\section{Introduction}

Histone acetylation in lysine residues is well-known for its critical roles in regulation of chromatin structure and function $[1,2]$. Recent studies indicate that, besides acetylation, histone lysine $(\mathrm{K})$ residues are also subjected to multiple types of short-chain acylation including malonylation, propionylation, butyrylation, crotonylation and succinylation [3-8]. The identification of these new

\footnotetext{
*These three authors contributed equally to this work.

Correspondence: Jianjun $\mathrm{Yu}^{\mathrm{a}}$, Jiemin Wong ${ }^{\mathrm{b}}$

${ }^{a} E-m a i l: y u j j 917 @ 163 . c o m$

${ }^{\mathrm{b}}$ E-mail: jmweng@bio.ecnu.edu.cn

Received 25 February 2017; revised 7 April 2017; accepted 10 April 2017; published online 12 May 2017
}

post-translational modifications (PTMs) has drastically expanded the repertoire of histone modifications and raises significant interest in exploring their distinct and/ or redundant functions in transcription as well as the enzymes for adding (writers) and removal (erasers), and proteins for reading (readers) these PTMs [3, 9].

Among the reported non-acetyl histone acylations, histone crotonylation is particularly interesting for the following reasons. First, it occurs broadly in all core histones and marks either active promoters or potential enhancers [4]. Second, histone crotonylation by p300 has been shown to promote transcription in vitro and manipulating cellular concentration of crotonyl-CoA affects gene expression [10]. Third, we show that a CBP/p300 mutant with defective histone acetyltransferase activity and competent crotonyltransferase activity is able to sub- 
stitute endogenous $\mathrm{CBP} / \mathrm{p} 300$ to promote $\mathrm{TGF} \beta$-induced transcriptional activation [54]. Fourth, two groups of histone acetylation readers previously identified have recently been shown to selectively recognize histone crotonylation over histone acetylation and other acylation [11-16]. These studies collectively support a potential non-redundant role for histone crotonylation vs histone acetylation. However, histone crotonylation is likely much less abundant compared to histone acetylation due to an approximately three orders of magnitude lower cellular concentration of crotonyl-CoA than acetyl-CoA [10]. Thus, the physiological relevance and functional significance of histone crotonylation remain to be demonstrated.

The identification of these new acylations in histones also raises the fundamental question on what enzymes or whether the previously identified histone deacetylases (HDACs and SIRTs) are responsible for the removal of these acylations. In this regard, previous studies have identified weak depropionylase and debutyrylase activities for several SIRTs [17, 18]. In addition, SIRT5 has been shown to possess robust desuccinylase, demalonylase and deglutarylase activities but poor deacetylase activity [19-22], leading to the suggestion that SIRTs have an expanded repertoire of deacylase activities. In agreement with this, SIRT1, SIRT2 and SIRT3 have been shown to exhibit widespread deacylase activity in vitro, although the deacylase activity is weaker in comparison to their deacetylase activity [23]. In addition, SIRT3 was shown to decrotonylate histones in living cells [24]. In contrast, by using recombinant proteins, both class I and II HDACs were shown to exhibit poor decrotonylase activities [4], although a weak decrotonylase activity was detected for HDAC3 in another study [25]. Thus, current studies on histone decrotonylases are primarily based on in vitro assays. Whether these HDCR activities are physiologically relevant and represent the major HDCR activities in mammalian cells remains to be determined. In addition, it is unknown if histone crotonylation is as dynamic as histone acetylation.

Here we demonstrate that class I HDACs are the major histone decrotonylases in mammalian cells and that histone crotonylation is as dynamic as histone acetylation. Notably, we have generated HDAC1 and HDAC3 mutants that maintain the HDCR activity but lose the HDAC activity. Using these mutants we demonstrate that histone crotonylation is not functionally redundant to histone acetylation but is critically important for general transcriptional regulation in mammalian cells. Furthermore, we demonstrate that mouse embryonic stem cells (ESCs) have a higher level of histone crotonylation than differentiated cells, and selective histone decrotonylation in ESCs results in cell differentiation.

\section{Results}

\section{Class I HDACs possess HDCR activity}

To identify cellular HDCR activity, we focused on the HDAC family and SIRT family HDACs since some of these proteins have been shown to possess deacylase activities. We ectopically expressed individual candidate protein in HeLa cells and performed immunofluorescent (IF) staining using a pan-crotonylated lysine (Kcr)-specific antibody that was well characterized in Supplementary information, Figure S1. There are three major advantages for this cell-based HDCR screening. First, ectopic overexpression could allow detection of even a weak enzymatic activity by IF staining. Second, ectopic overexpression could allow the detection of candidate enzyme(s) whose activity is highly dependent on the complex formation. Third, IF staining allows side-byside analysis of enzymatic activity based on a large number of transfected cells vs untransfected control cells. As a positive control, we also performed IF staining using an anti-pan acetylated lysine (Kac) antibody. Using this approach, we detected a robust HDCR activity for all class I HDAC members HDAC1, HDAC2, HDAC3 and HDAC8 (Figure 1A). As expected, these HDACs also exhibited a robust HDAC activity (Figure 1A). However, as shown in Figure 1B and Supplementary information, Figure S2A, under the same experimental conditions we failed to detect any significant HDCR activity for all class II HDACs. The lack of HDCR activity observed for class II HDACs was unlikely due to problems in protein expression, as HDAC activity was observed for each of the proteins except for HDAC10 (Figure 1B and Supplementary information, Figure S2A). In addition, no HDCR activity was detected for HDAC11, the sole member of class IV HDAC (Figure 1C). Thus, our cellbased activity assay revealed a robust HDCR activity for all class I but none of class II and class IV HDACs.

Among the SIRT family deacetylases, we found that SIRT1 displayed both HDAC and HDCR activities in cells (Figure 1D), in agreement with its reported HDCR activity in vitro [23]. However, we failed to detect HDCR activity for SIRT2, SIRT3, SIRT4, SIRT5 and SIRT7 (Figure 1D and Supplementary information, Figure S2B). The lack of HDCR activity for both SIRT2 and SIRT3 in our cell-based assay was further substantiated by IF staining using seven different histone lysine-specific crotonylation antibodies (Supplementary information, Figure S3A). Furthermore, while we detected by WB analysis a substantially reduced level of histone acetylation upon ectopic expression of SIRT2 or SIRT3 

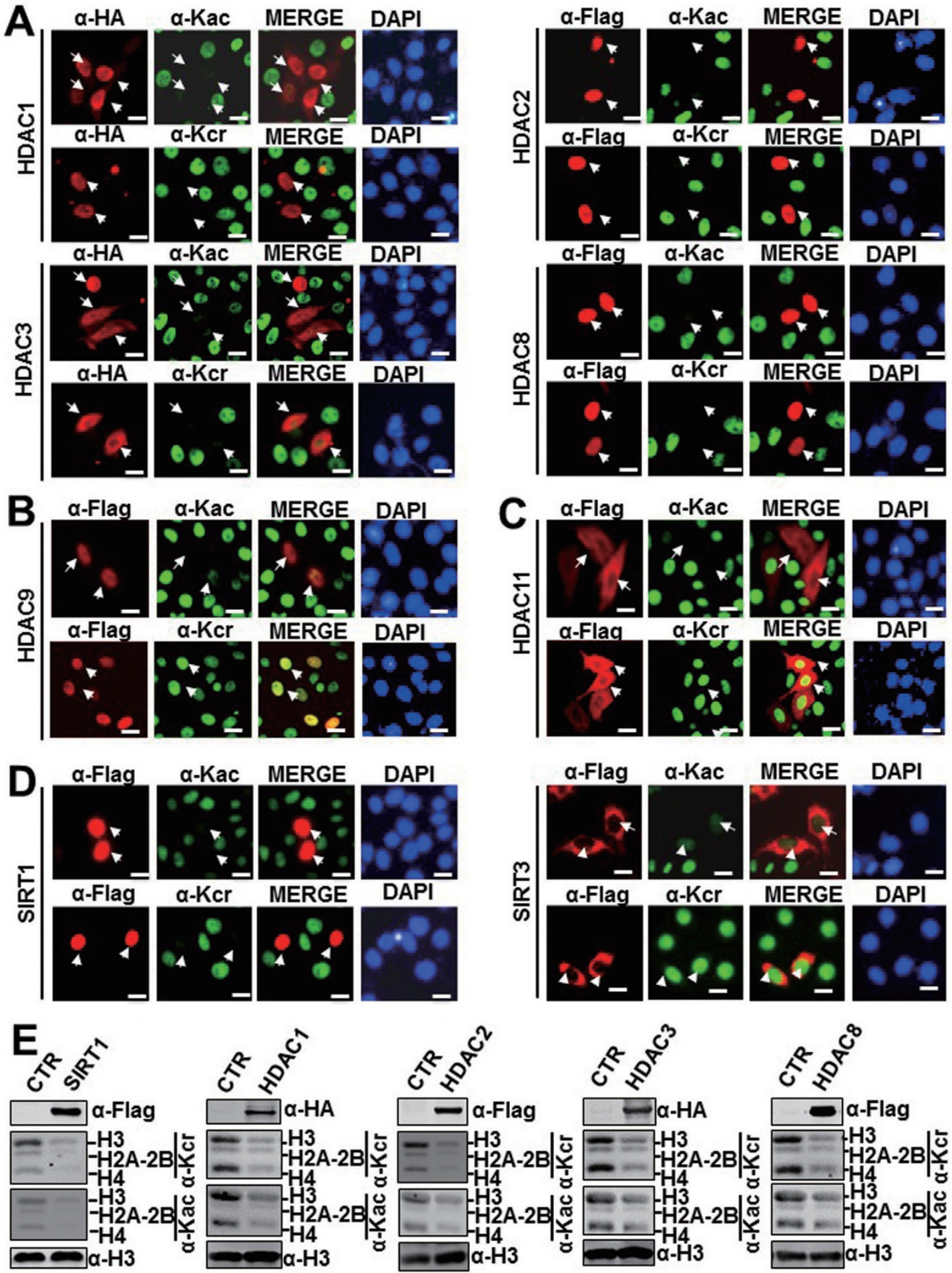

Figure 1 Class I HDACs possess HDCR activity. (A) Examining the HDCR activity for ectopically expressed class I HDACs by IF staining using pan-Kcr antibody. HeLa cells were transfected with expression plasmids for HA-tagged HDAC1 and HDAC3 and Flag-tagged, HDAC2 and HDAC8, respectively, and the HDCR and HDAC activities were detected by immunostaining using pan-Kcr or pan-Kac antibody as indicated. Scale bar, $20 \mu \mathrm{m}$. (B-C) Examining the HDCR activity for ectopically expressed class II HDAC9 (B) and class IV HDAC11 (C) as above. (D) Examining the HDCR activity for ectopically expressed SIRTs as above. (E) WB analysis of core histones derived from control and HeLa cells transfected with SIRT1 or indicated class I HDAC using antibodies as indicated. Also shown were WB data of whole-cell extracts showing the levels of ectopically expressed SIRT1 and individual class I HDACs. 
in $293 \mathrm{~T}$ cells, we did not observe any significant change in histone crotonylation by WB analysis using a pan-Kcr antibody and several site-specific histone Kcr antibodies (Supplementary information, Figure S3B).

To validate our surprising finding that class I HDACs possess robust HDCR activity, we ectopically expressed each class I HDAC in 293 T cells by high efficiency transfection. Subsequent WB analysis of core histones revealed that expression of each class I HDAC resulted in substantially reduced histone acetylation as well as histone crotonylation (Figure 1E). The same WB analysis also confirmed an HDCR activity for SIRT1. Notably, WB analysis using the pan-Kcr antibody revealed that SIRT1 and class I HDACs were able to decrotonylate $\mathrm{H} 3, \mathrm{H} 2 \mathrm{~A} / \mathrm{H} 2 \mathrm{~B}$ and $\mathrm{H} 4$ (Figure 1E).

In sum, our cell-based assays revealed a robust HDCR activity for class I HDACs and SIRT1. On the other hand, our cell-based assays revealed poor, if any, HDCR activity for class II and class IV HDACs, and other members of SIRT proteins including SIRT2 and SIRT3.

Class I HDACs are major cellular histone decrotonylases

Having demonstrated the HDCR activity of class I HDACs and SIRT1, we next assessed if they represent the major HDCR activity in cells. To this end, we performed RNA interference experiments to knock down SIRT1, HDAC1, HDAC2 and HDAC3, respectively, in HeLa cells and prepared core histones by acid extraction. Subsequent WB analyses revealed that knockdown of HDAC1, HDAC2 or HDAC3 led to increased levels of histone crotonylation as well as acetylation (Figure 2A). Furthermore, simultaneous knockdown of HDAC1/2/3 led to a more robust increase of both histone crotonylation and acetylation (Figure 2A). On the other hand, knockdown of either SIRT 1 or SIRT3 alone or SIRT1/3/5 in combination did not obviously affect the global levels of histone crotonylation as well as acetylation (Supplementary information, Figure S4A). The efficient knockdown of HDACs or SIRTs by their corresponding siRNA was verified by WB (Figure 2A) or quantitative reverse transcription PCR (qRT-PCR) analysis (Supplementary information, Figure S4B). As knockdown of class I HDACs, especially in combination, led to a substantial increase of histone crotonylation, these results provide evidence that class I HDACs represent the major HDCR activity in cells.

To further assess if class I HDACs but not SIRT proteins are responsible for bulk histone decrotonylation in mammalian cells, we treated HeLa cells with either the HDAC-specific inhibitor Trichostatin A (TSA) or the SIRT-specific inhibitor nicotinamide (NAM) overnight and examined the effect on histone acetylation and cro- tonylation by IF staining analysis. The representative results in Figure 2B showed that, while NAM treatment led to a modest increase of crotonylation and acetylation, TSA treatment led to a more robust increase of crotonylation and acetylation (Figure 2B). To assess in more detail the differential effect of NAM and TSA on histone crotonylation, we treated HeLa cells with NAM and TSA for various times. Subsequent WB analysis in Figure $2 \mathrm{C}$ showed that TSA treatment led to a gradual increase of histone crotonylation over a 24-h period of time and a substantial increase of histone crotonylation could be observed as early as $3 \mathrm{~h}$ post treatment. Comparison of histone acetylation and crotonylation revealed a similar kinetics in response to TSA. The same results were observed when the experiments were performed with NIH3T3 (Figure 2D), 293T, A549 and HCT116 cells (Supplementary information, Figure S5). Together, these data suggest that class I HDACs represent the major HDCR activity in cells. Furthermore, these data indicate that histone crotonylation is as dynamic as acetylation.

Site specificity of histone decrotonylation by various histone decrotonylases

Having established that SIRT1 and class I HDACs possess the HDCR activity, we next examined their site specificity in histone decrotonylation. We analyzed this first by IF staining using a panel of site-specific histone Kcr antibodies characterized in a previous study [4]. As shown in Figure 3A, we found HDAC1 is active for each of the sites tested, namely $\mathrm{H} 3 \mathrm{~K} 4 \mathrm{cr}$, H3K $9 \mathrm{cr}, \mathrm{H} 3 \mathrm{~K} 23 \mathrm{cr}$, $\mathrm{H} 4 \mathrm{~K} 8 \mathrm{cr}$ and H4K12cr. However, this is not the case for SIRT1, since ectopic expression of SIRT1 only led to a marked reduction of $\mathrm{H} 3 \mathrm{~K} 9 \mathrm{cr}$ and $\mathrm{H} 4 \mathrm{~K} 8 \mathrm{cr}$, and a moderate reduction of $\mathrm{H} 3 \mathrm{~K} 4 \mathrm{cr}$ and $\mathrm{H} 3 \mathrm{~K} 18 \mathrm{cr}$ (Figure $3 \mathrm{~B}$ and $3 \mathrm{C})$. The difference in site specificity also exists among members of class I HDACs and is best illustrated by the results of WB analysis in Figure 3D-3G. For example, HDAC2, HDAC3 and HDAC8 were essentially inactive for decrotonylation of $\mathrm{H} 3 \mathrm{~K} 23 \mathrm{cr}$, and $\mathrm{HDAC} 2$ and HDAC3 were also poorly active for $\mathrm{H} 3 \mathrm{~K} 4 \mathrm{cr}$ and $\mathrm{H} 4 \mathrm{~K}$ $12 \mathrm{cr}$ (Figure 3D-3G). Together, these data demonstrate that SIRT1 and class I HDACs are histone decrotonylases with distinct site specificity.

\section{The same catalytic center for both HDAC and HDCR ac-} tivities

Our data clearly demonstrate that SIRT1 and class I HDACs possess both HDAC and HDCR activities. We next tested if the same enzymatic center is responsible for both activities. As shown in Supplementary information, Figure S6A for SIRT1 by IF staining, the HDCR activity for SIRT1 could be inhibited by NAM but not 

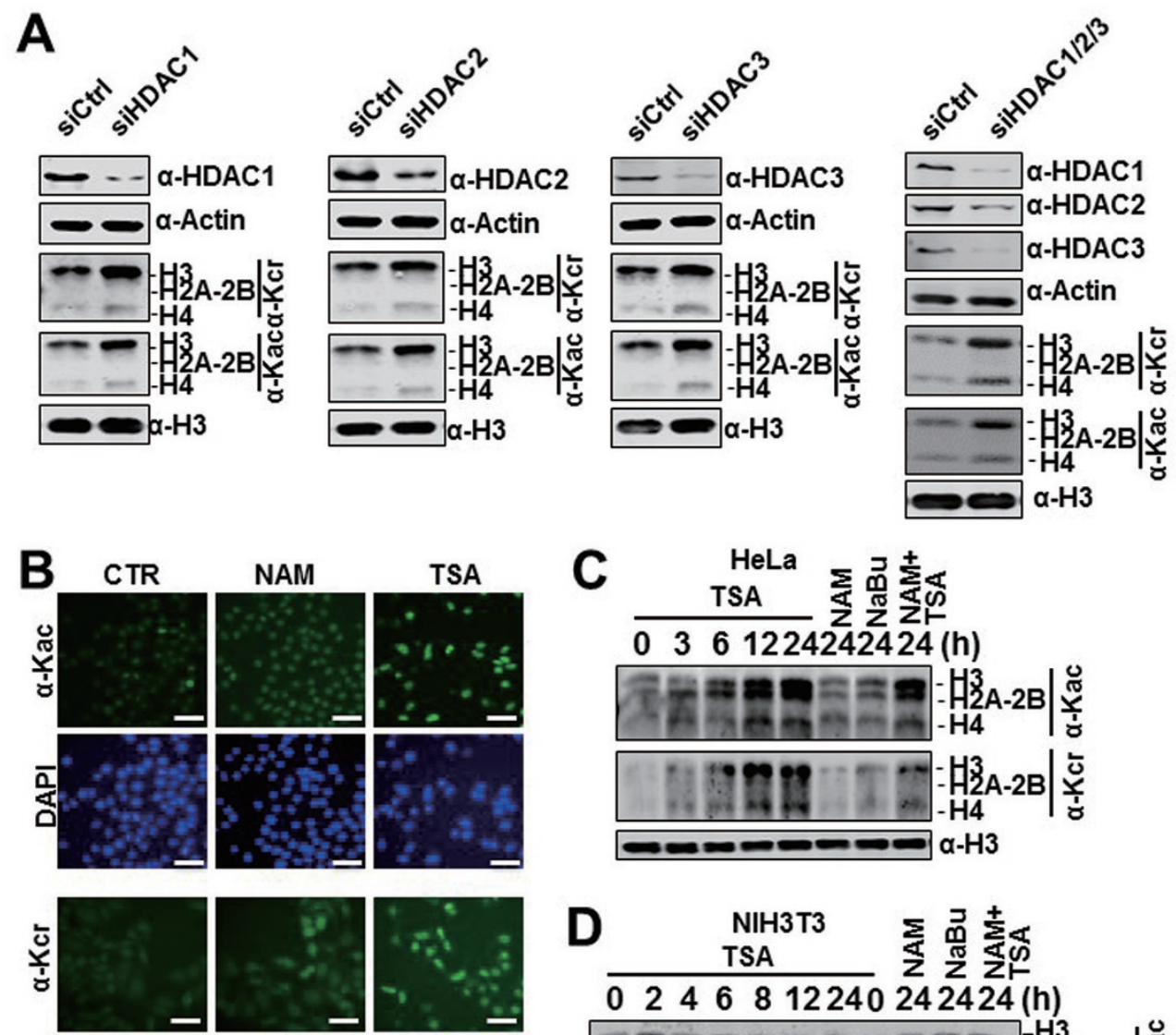

TSA

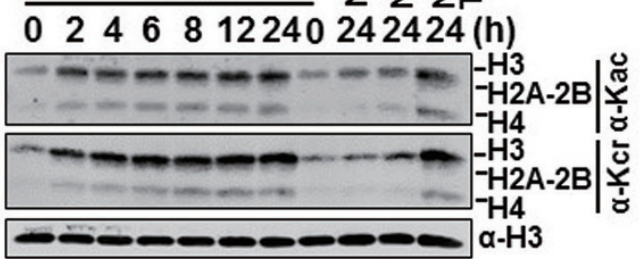

Figure 2 Class I HDACs are the major cellular histone decrotonylases responsible for dynamic histone decrotonylation. (A) WB analysis showing that knockdown of HDAC1, HDAC2 and HDAC3 individually or in combination resulted in elevated levels of histone crotonylation as well as acetylation. HeLa cells were treated with siRNAs against class I HDAC as indicated for 3 days before subjected to WB analysis. The efficiency of siRNA knockdown was verified by WB. (B) IF assay showing the differential effect of TSA and NAM treatment on cellular acetylation and crotonylation. HeLa cells were treated with $1 \mu \mathrm{M}$ TSA or $5 \mathrm{mM}$ NAM for $12 \mathrm{~h}$ before IF staining. Scale bar, $60 \mu \mathrm{m}$. (C-D) HeLa cells (C) and NIH3T3 cells (D) were treated with TSA, NAM, NaBu (sodium butyrate) or $\mathrm{NAM}^{+}$TSA for various times as indicated and core histones were prepared and subjected to WB analysis using antibodies as indicated. TSA, $1 \mu \mathrm{M}$; NAM, $5 \mathrm{mM}$ and NaBu, $5 \mathrm{mM}$.

TSA. Similarly, the HDCR activity for HDAC1 could be effectively inhibited by TSA but not NAM (Supplementary information, Figure S6B), suggesting that the same catalytic center is responsible for both HDCR and HDAC activities. In support of this idea, Supplementary information, Figure S6C shows that a previously reported SIRT1 mutant that is inactive in HDAC activity is also inactive in histone decrotonylation [26]. Similarly two HDAC1 mutants defective in HDAC activity were also found to be impaired for HDCR activity (Supplementary information, Figure S6C). Thus, the HDCR activity is intrinsic to SIRT1 and class I HDACs, and the same catalytic center is involved in both histone deacetylation and decrotonylation.

Generation of novel HDAC1/3 mutants with HDCR but impaired HDAC activity

Although the previous in vitro study and our unpublished data with HAT-deficient and HCR-competent CBP/p300 mutants [54] demonstrate that histone crotonylation can promote transcriptional activation [10], a central question is whether histone crotonylation has a 


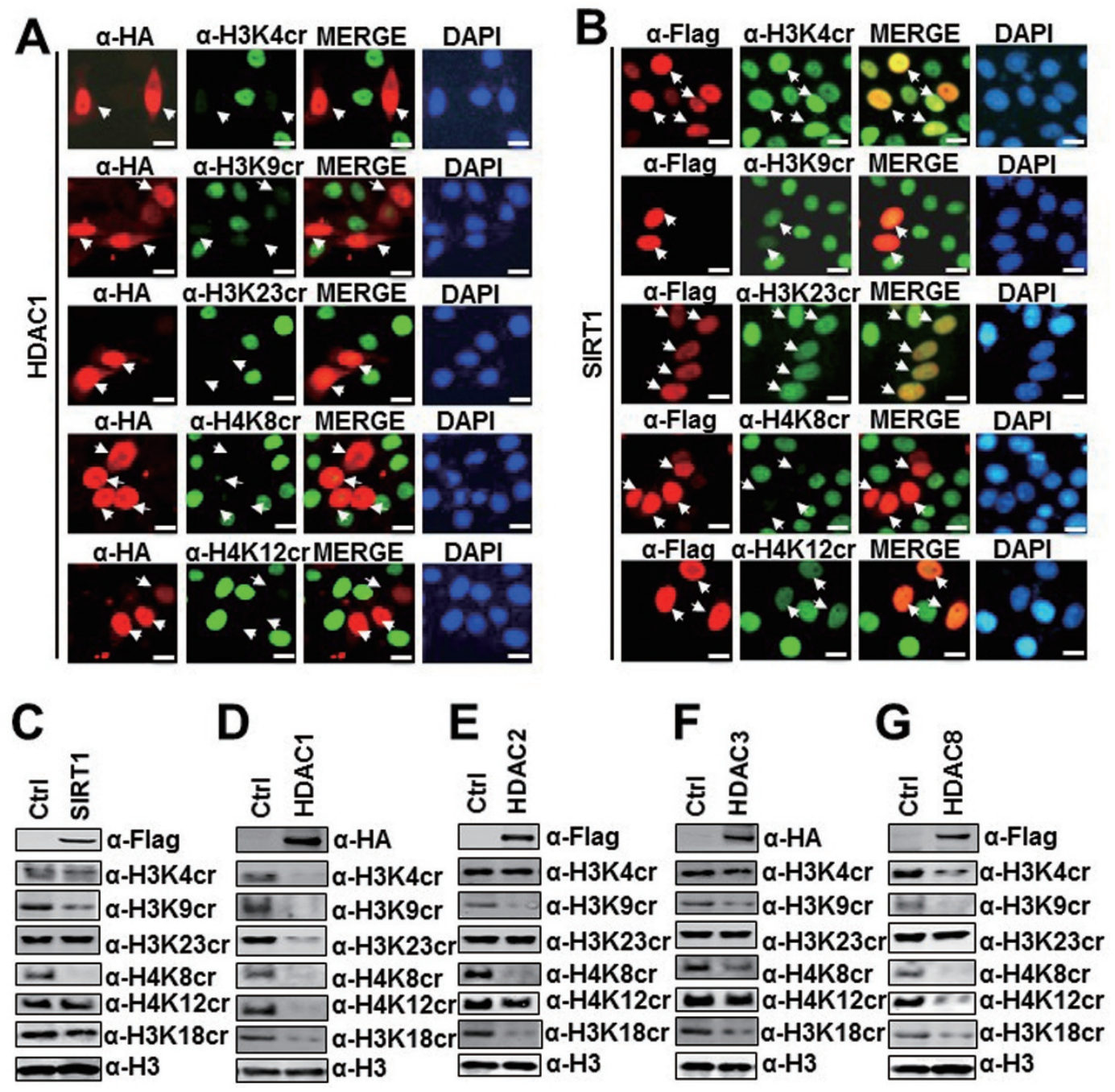

Figure 3 SIRT1 and class I HDACs show distinct site specificity for histone decrotonylation. (A-B) Examining the site specificity of histone decrotonylation by HDAC1 (A) and SIRT1 (B) by IF assay using various site-specific histone crotonylation antibodies. HeLa cells were transfected with expression plasmids for HA-HDAC1 or Flag-SIRT1, and IF staining was performed 2 days after transfection. Scale bar, $15 \mu \mathrm{m}$. (C-G) Examining the site specificity of histone decrotonylation by SIRT1 (C), HDAC1 (D), HDAC2 (E), HDAC3 (F) and HDAC8 (G) via WB analysis using various site-specific histone crotonylation antibodies as indicated. HeLa cells were transfected with expression plasmids as indicated for 2 days before subjected to core histone preparation and WB analysis.

general or specific role in transcriptional activation under the physiological condition. To address this crucial question on histone crotonylation, we aimed to screen for an HDAC mutant with competent HDCR but impaired HDAC activity. Given HDAC1 being the most active HDCR in our assays, we initially focused our screening on various HDAC1 catalytic center mutants. Despite our great effort, we obtained only the mutants that either lost both activities (Supplementary information, Figure S7) or had moderate to no effect on both activities (data not shown). We then took a different approach. Given that class II HDACs are deficient in HDCR activity, we compared the sequences between class I and class II HDACs, and identified the major differences between them (Figure 4A). We then swapped the amino acids AGG in HDAC1 with VRPP in class II HDACs, expecting to generate an HDAC1 mutant possessing HDAC but losing HDCR activity, as such sequence exchange would predict to make the pocket for substrate narrower in HDAC1 (Figure 4B). To our surprise, we found this mutant HDAC1 (HDAC1VRPP) was inactive for HDAC but active for HDCR activity (Figure 4C). As shown in Supplementary infor- 
A
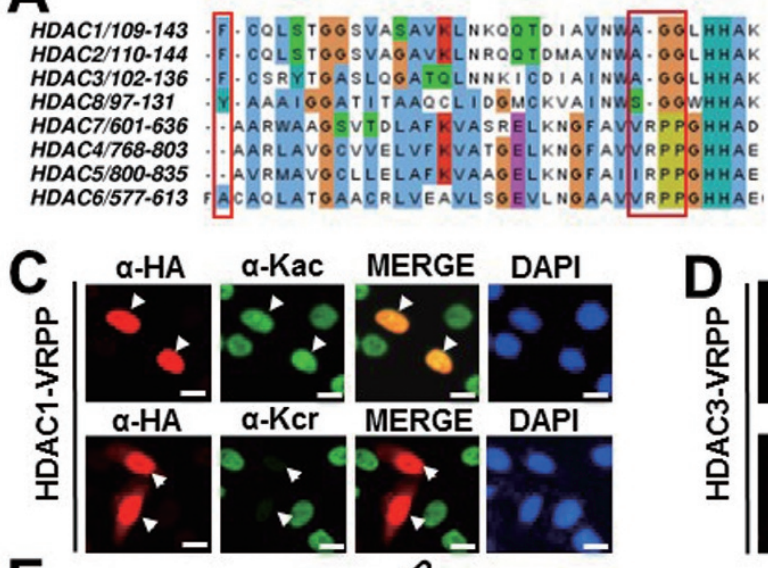

E

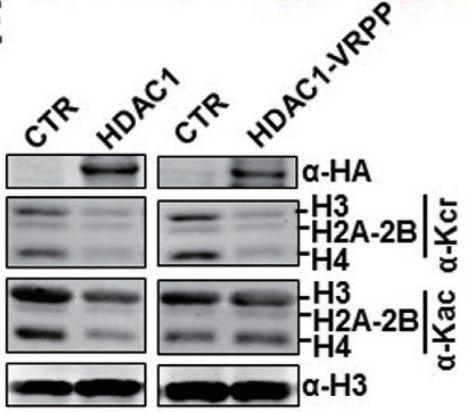

G

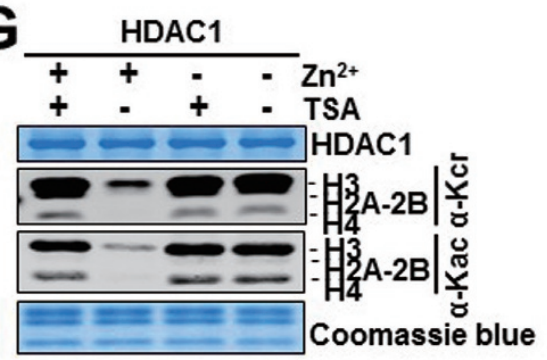

H

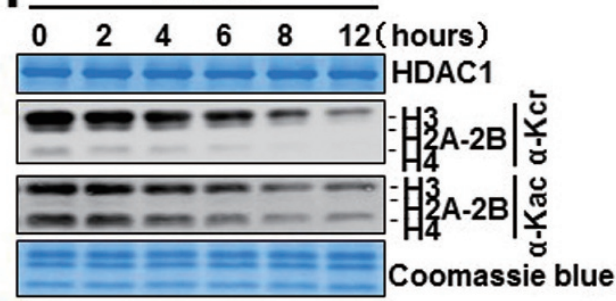

B
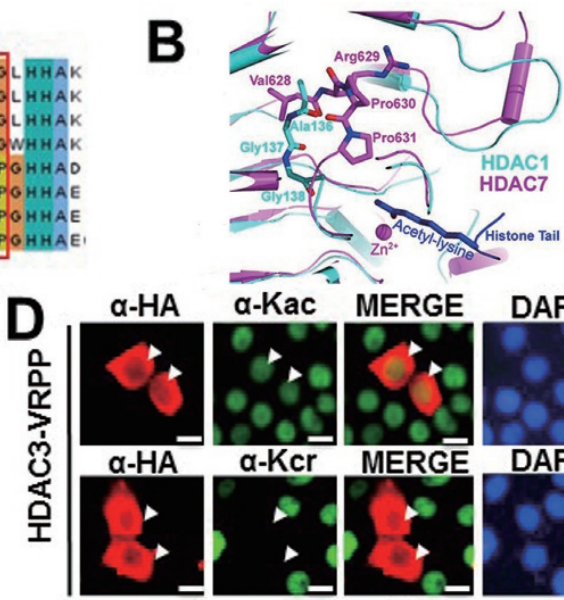

$\mathbf{F}$

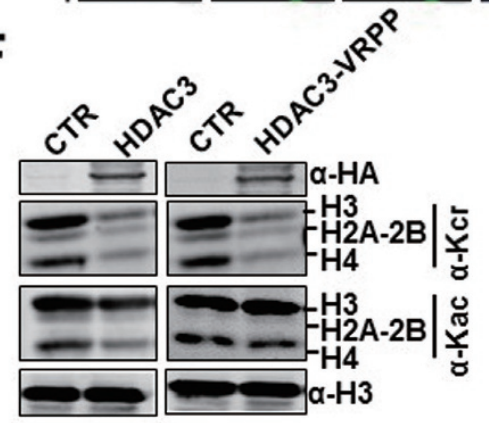

I

HDAC1-VRPP
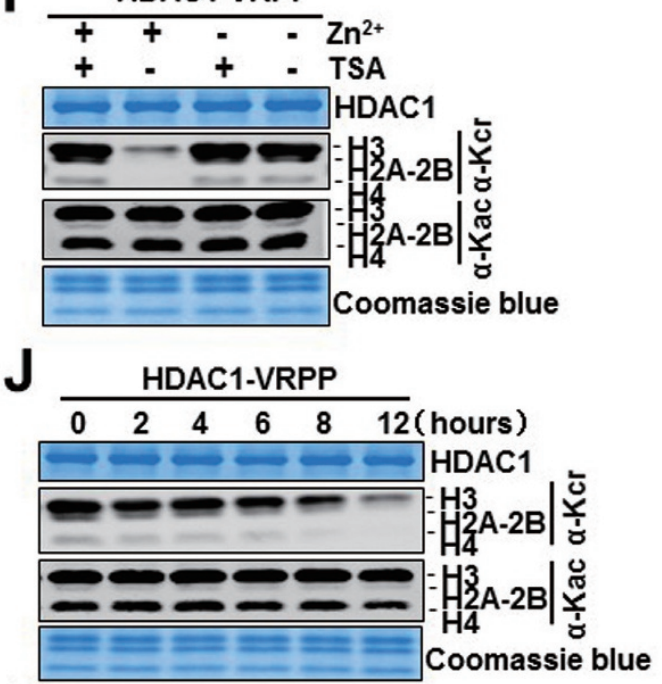

Figure 4 Identification and characterization of HDAC1 and HDAC3 mutants that are inactive for HDAC but active for HDCR activity. (A) Sequence alignments comparing the key differences between class I and class II HDACs. (B) Diagram illustrating the potential structural differences between class I and class II HDACs. The structures used: HDAC1 (PDB 4BKX, cyan) [51], HDAC7 (PDB 3C0Z, magenta) [52]; note that the acetyl lysine of histone tail was from HDAC8 complex (PDB 2V5W, blue) [53]. (C-D) IF analysis showing that the HDAC1-VRPP mutant (C) and the HDAC3-VRPP mutant (D) were inactive for HDAC activity but active for HDCR activity. HeLa cells were transfected with expression plasmids for HA-HDAC1-VRPP or HA-HDAC3VRPP mutant and IF staining was performed 2 days after transfection. Scale bar, $15 \mu \mathrm{m}$. (E-F) WB analysis showing that the HDAC1-VRPP mutant (E) and HDAC3-VRPP mutant (F) were active for HDCR activity but inactive for HDAC activity. HeLa cells were transfected with expression plasmids for WT or mutant HDAC1 or HDAC3 as indicated. Core histones were prepared and WB analysis was performed with 2 days after transfection. (G-H) In vitro HDAC and HDCR activities were assayed for wild-type HDAC1 with or without addition of $\mathrm{Zn}^{2+}$ or TSA as indicated for $12 \mathrm{~h}(\mathbf{G})$ or with various times (H). HA-HDAC1 proteins were purified from HeLa cells and core histones were also prepared from HeLa cells. TSA, $1 \mu$ M. (I-J) In vitro HDAC and HDCR activities were assayed for the HDAC1-VRPP mutant as described above. 
mation, Figure S8, this mutant exhibited the same site specificity for histone decrotonylation as the wild-type HDAC1. Furthermore, an equivalent HDAC3 mutant (HDAC3-VRPP) was also inactive for HDAC but active for HDCR activity (Figure 4D). The loss of HDAC activity for these HDAC1 and HDAC3 mutants was clearly substantiated by WB analysis of core histone proteins derived from HeLa cells overexpressing WT or mutant HDAC1 (Figure 4E) and WT or mutant HDAC3 proteins (Figure 4F).

To further characterize the novel property of HDAC1VRPP mutant, we carried out in vitro histone decrotonylation and deacetylation assays using immunoaffinity-purified WT and mutant HDAC1. We first established that the purified WT HDAC1 exhibited robust activity to decrotonylate and deacetylate core histone substrates in a $\mathrm{Zn}^{2+}$-dependent and TSA-sensitive manner (Figure 4G). These HDCR and HDAC activities were further validated in a time course experiment, showing progressive decrotonylation and deacetylation with prolonged reactions (Figure 4H). Under the same conditions, we found that the HDAC1-VRPP mutant was active for histone decrotonylation but completely inactive for histone deacetylation (Figure 4I and 4J). Thus, both in vivo and in vitro assays demonstrated that the novel HDAC1-VRPP mutant has impaired HDAC but maintains an active HDCR activity. Although how swapping of the AGG sequence with VRPP leads to generation of these novel mutants awaits structural study, the availability of these mutants provides us with the opportunity to directly assess the effect of histone decrotonylation in transcriptional regulation.

The HDCR activity is sufficient to repress transcription in reporter assay

We first tested if the HDAC1-VRPP mutant is able to repress transcription in reporter assays. We constructed Gal4-DBD fusion proteins of wild-type HDAC1, H141A and VRPP mutants, and assayed their ability to repress transcription from a $5 \times$ UAS-TK luciferase reporter. As shown in Supplementary information, Figure S9A, we found the H141A mutant with defect in both HDAC and HDCR activities was unable to repress transcription, whereas both wild-type HDAC1 and the HDAC1VRPP mutant were able to repress transcription. We also compared the ability for wild-type, H141A and VRPP mutants to repress transcriptional activation induced by various transcription factors in reporter assays. While co-transfection of wild-type HDAC1 repressed transcriptional activation by Sox2, co-transfection of the HDAC1VRPP mutant also led to transcriptional repression. This repression activity is dependent on the HDCR activity of
HDAC1, as H141A mutant defective in both HDCR and HDAC activities failed to do so (Supplementary information, Figure S9B). Similarly, we found the HDAC1VRPP mutant was able to repress transcriptional activation by Oct4 (Supplementary information, Figure S9C) and SMAD3 (Supplementary information, Figure S9D). Since the HDAC-VRPP mutant is defective in deacetylation but active in decrotonylation, these results provide first evidence that decrotonylation without deacetylation is sufficient to repress transcription.

\section{Histone decrotonylation results in global transcriptional repression}

To better assess the role of histone crotonylation in transcriptional regulation, we generated stable HeLa cell lines in which the expression of wild-type HDAC1 or the HDAC1-VRPP mutant (without tag) is tetracycline inducible. As shown in Figure 5A, treatment with doxycycline (Dox), an analog of tetracycline, induced the expression of both wild-type and the HDAC1-VRPP mutant by 2-3-folds over the level of endogenous HDAC1 within $48 \mathrm{~h}$. Importantly, while induced expression of wild-type HDAC1 resulted in a global reduction of both histone acetylation and crotonylation at $36 \mathrm{~h}$, induced expression of the HDAC1-VRPP mutant only led to reduced levels of histone crotonylation but not acetylation (Figure 5A), further confirming that the HDAC1-VRPP mutant is active in histone decrotonylation but inactive in histone deacetylation. A 36-h Dox treatment was thus chosen for analyzing the effect of decrotonylation on global transcription because it was more effective in inducing histone decrotonylation. We first prepared total RNAs from an equal number of control, wild-type and HDAC1-VRPP-inducible cells treated with or without Dox for 36 h. As shown in Figure 5B, induced expression of both wild-type and HDAC1-VRPP resulted in reduced levels of total RNA, suggesting that, like induced wild-type HDAC1, the induced HDAC1-VRPP mutant was capable of repressing global transcription. To test this further, we analyzed the effect on transcription of six housekeeping genes by qRT-PCR. As shown in Figure $5 \mathrm{C}$, induced expression of wild-type HDAC1 or the HDAC1-VRPP mutant resulted in reduced expression of all six housekeeping genes, suggesting that the HDAC1VRPP mutant is as active as wild-type HDAC1 for transcriptional repression.

As reported in several recent studies [27, 28], addition of a spike-in internal control allows comparison of global- and gene-specific levels of transcription among different samples by RNA-seq analysis. In order to analyze more precisely the effect of selective histone decrotonylation on transcription, we thus added spike-in yeast 

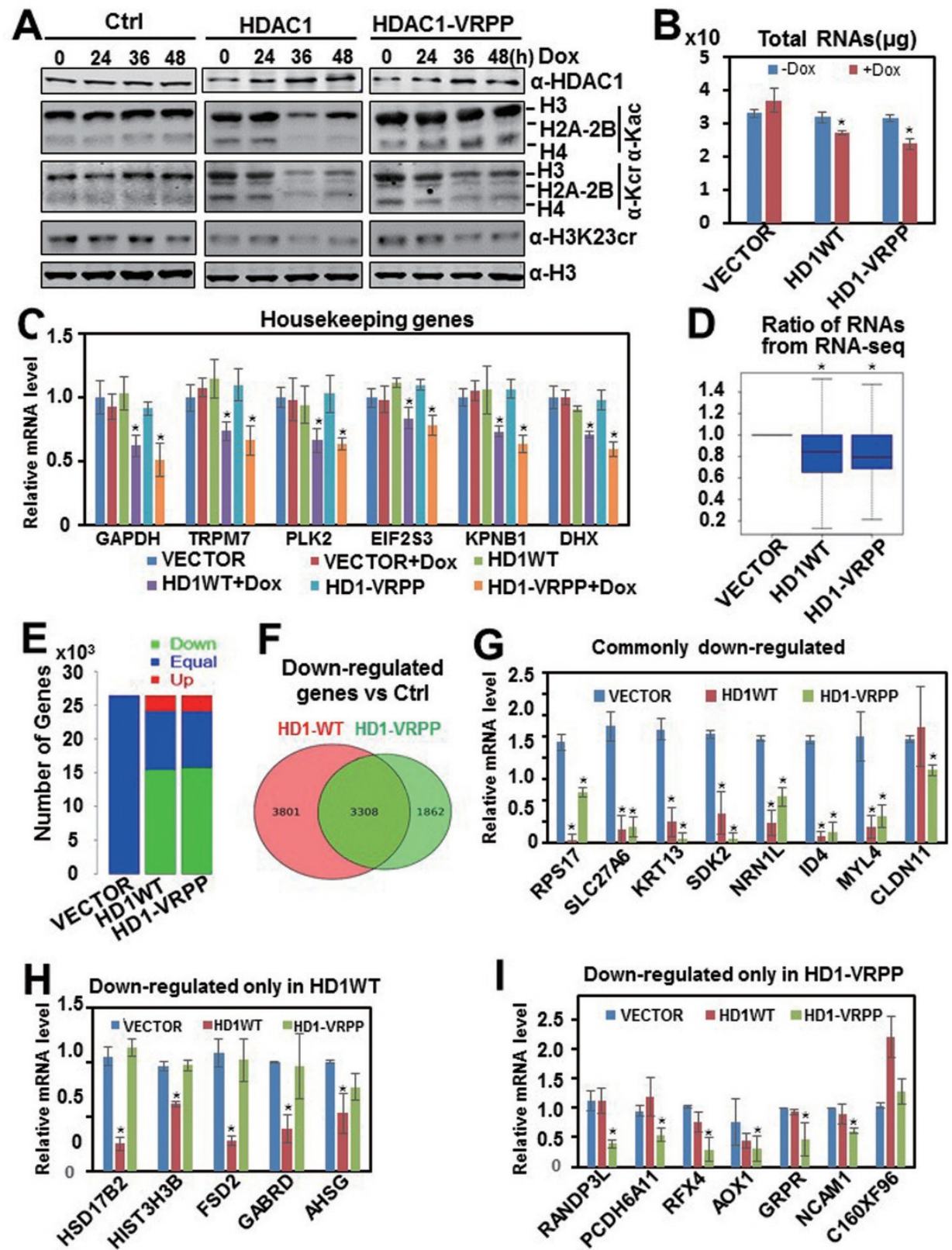

Figure 5 Selective histone decrotonylation results in global transcriptional repression. (A) WB analysis showing the effect of Dox-induced expression of wild-type HDAC1 and the HDAC1-VRPP mutant on histone acetylation and crotonylation. Note that the induced expression of HDAC1 resulted in both histone deacetylation and decrotonylation, whereas induced expression of HDAC1-VRPP resulted in only reduced histone crotonylation. (B) Measurement of total levels of RNAs from the same numbers of cells $\left(5 \times 10^{6}\right)$ with or without induced expression of HDAC1 and HDAC1-VRPP. Data as mean of total RNAs ( $\left.\mu \mathrm{g}\right)$ $\pm \mathrm{SD}$ of three technical duplicates. $P \leq 0.05$. (C) qRT-PCR analysis of six housekeeping genes for their levels of transcription in control HeLa or HeLa cells with or without induced expression of HDAC1 and HDAC1-VRPP. Data as relative levels of mRNA to the control \pm SD of three technical duplicates. $P \leq 0.05$. (D) The ratio of total RNAs determined on the basis of RNA-seq analysis of the RNA samples from the same number of control, wild-type HDAC1 and HDAC1-VRPP mutant cells induced with Dox for $36 \mathrm{~h}$. The yeast RNAs were added to the samples as a spike-in RNA control for normalization after RNA-seq. (E) The RNA-seq data were analyzed for the genes with upregulated, no change or downregulated expression in the Dox-treated wild-type HDAC1 or HDAC1-VRPP cells in comparison to the control cells. (F) Diagram showing the genes common downregulated, downregulated in wild-type HDAC1 only and downregulated in HDAC1-VRPP cells only. (G-I) qRTPCR analysis examining the eight randomly selected commonly downregulated genes (G), five downregulated only in the wild-type HDAC1 expressing cells $\mathbf{( H )}$ and seven downregulated only in the HDAC1-VRPP expressing cells (I). Data are represented as relative level of transcription to the control $\pm \mathrm{SD}$ of technical replicates. $P \leq 0.05$. 
total RNAs as an internal control to total RNA samples prepared from the equal number of the Dox-induced control, wild-type and mutant HDAC1 cells before samples were processed for RNA-seq analysis. As shown in Figure 5D, the RNA-seq reads after normalization to the internal spike-in control revealed that the levels of total mRNAs were decreased by an average of $\sim 16 \%$ upon induction of wild-type HDAC 1 and by $22 \%$ upon induction of the mutant HDAC1. Furthermore, in comparison to the transcript profile in the control cells, induction of mutant HDAC1 had a similar impact on gene expression as induction of wild-type HDAC1, resulting in reduced expression for $>15000$ genes and elevated expression for $<2000$ genes, respectively (Figure 5E). By using 1.5fold change as a cutoff, induction of wild-type HDAC1 led to downregulation of 7109 genes and upregulation of 1105 genes, whereas induction of mutant HDAC1 led to downregulation of 5170 genes and upregulation of 1417 genes (Figure 5F). We did not analyze further the upregulated genes because they are more likely the indirect effect of induced wild-type and mutant HDAC1. The downregulated genes were further categorized into three groups: commonly downregulated genes, downregulated by WT HDAC1 only and downregulated by mutant HDAC1 only (Figure 5F). These three groups of genes were validated by qRT-PCR analysis. As shown in Figure $5 \mathrm{G}$, seven of the eight commonly downregulated genes were confirmed to be downregulated upon induction of either wild-type or mutant HDAC1. In addition, we selected five genes downregulated only in the wild-type HDAC1-induced cells and seven genes downregulated only in the HDAC1-VRPP-induced cells for verification by qRT-PCR, and the results in Figure 5H and 5I in general confirmed their differential expression status. Together these results indicate that selective histone decrotonylation could repress global transcription to a similar extent as both histone deacetylation and decrotonylation. In addition, these results indicate that a subset of genes could be differentially regulated by histone crotonylation or histone acetylation.

Promoter histone decrotonylation correlates with transcription repression

Having observed that induced expression of WT or HDAC1-VRPP mutant repressed both common and unique genes, we next analyzed by chromatin immunoprecipitation (ChIP) assay if repression by HDAC-VRPP correlates directly with histone decrotonylation at the promoter region. Using a previously reported anti-H3K$18 \mathrm{cr}$ antibody $[4,10]$, we found that induced expression of either WT or mutant HDAC1 led to reduced levels of $\mathrm{H} 3 \mathrm{~K} 18 \mathrm{cr}$ on the promoter regions of four commonly downregulated genes tested (Figure 6A). However, for three genes whose transcription was downregulated only in WT HDAC1-expressing cells, reduction of $\mathrm{H} 3 \mathrm{~K} 18 \mathrm{cr}$ was clearly less in the mutant HDAC1-expressing cells (Figure 6B). On the contrary, for four genes which were downregulated only in the mutant HDAC1-expressing cells, reduction of $\mathrm{H} 3 \mathrm{~K} 18 \mathrm{cr}$ was more dramatic in the mutant HDAC1-expressing cells (Figure 6C). In addition, ChIP results demonstrated that induced expression of wild-type HDAC1 led to consistently reduced levels of $\mathrm{H} 4 \mathrm{ac}$ for genes commonly downregulated (Figure $6 \mathrm{~A}$, lower panel) and genes uniquely downregulated by WT (Figure 6B, lower panel) but not for genes uniquely downregulated by mutant (Figure 6C, lower panel). Consistent with a lack of HDAC activity for mutant HDAC1, induced expression of the HDAC1-VRPP mutant had no effect on H4ac (Figure 6A-6C, lower panel). Collectively these data indicate that histone decrotonylation in the target gene promoters correlates with transcriptional repression by the HDAC1-VRPP mutant.

\section{Histone decrotonylation selectively impairs promoter re- cruitment of crotonylation reader proteins}

The selective histone decrotonylation by the HDAC1VRPP mutant provides us an opportunity to test if the newly identified histone crotonylation reader proteins associate with gene promoters in a crotonylation-dependent manner. To this end, we derived from the inducible HDAC1-VRPP HeLa cell line a pool of cells stably expressing Flag-tagged AF9, a YEATS domain crotonylation reader protein [11]. The cells were treated without or with Dox to induce HDAC1-VRPP expression and the association of Flag-AF9 with gene promoters was analyzed by ChIP assay. We found that Dox treatment substantially reduced the levels of Flag-AF9 associated with the promoters of genes commonly or uniquely downregulated by HDAC-VRPP but not the genes downregulated only by WT HDAC1 (Figure 6D). To test whether selective histone decrotonylation also affects the promoter association of double PHD type crotonylation readers [12], we ectopically expressed Flag-DPF2 in the inducible HDAC1-VRPP cells and carried out ChIP assay as described above. As a control, we also ectopically expressed BRD4 [29, 30], a histone acetylation reader, in the inducible HDAC1-VRPP cells and carried out ChIP assay. As shown in Figure 6E, we found that, much like Flag-AF9, induction of HDAC-VRPP expression substantially reduced the levels of Flag-DPF2 on the promoters of genes commonly or uniquely downregulated by HDAC-VRPP but not genes downregulated only by WT HDAC1 (Figure 6E). On the other hand, ChIP results revealed that induction of HDAC1-VRPP did not 

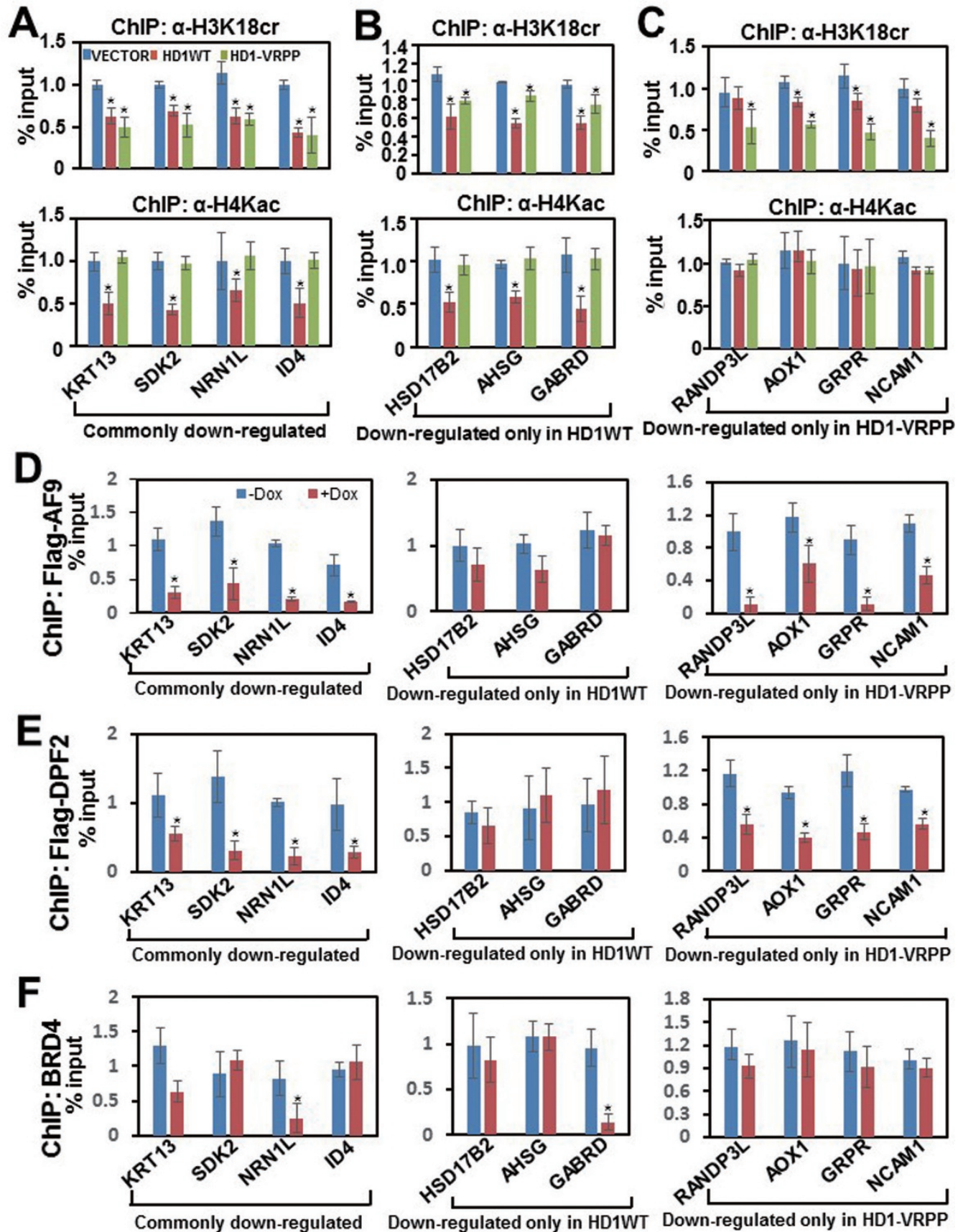

Figure 6 Transcriptional repression by the HDAC-VRPP mutant correlates with promoter histone decrotonylation and diminished promoter association of crotonylation reader proteins. (A-C) ChIP assays were performed with Dox-treated control, WT HDAC-1-inducible and HDAC1-VRPP-inducible HeLa cells to compare the status of histone crotonylation (H3K18cr) and histone acetylation ( $\mathrm{H} 4 \mathrm{ac}$ ) at the promoter regions for the commonly downregulated genes (A), downregulated only in WT HDAC1-expressing cells (B), and downregulated only in HDAC1-VRPP-expressing cells (C). H4ac was determined with an antibody against $\mathrm{K} 5 / \mathrm{K} 8 / \mathrm{K} 12 / \mathrm{K} 16$-acetylated $\mathrm{H} 4$. Data are represented as means of $\%$ input $\pm \mathrm{SD}$ of technical replicates. $P \leq$ 0.05. (D) ChIP assays were performed with stable Flag-AF9 expressing HDAC-1-VRPP-inducible cells treated with or without Dox for $36 \mathrm{~h}$ using anti-Flag antibody as above. Data are represented as means of \% input \pm SD of technical replicates. $P \leq$ 0.05. (E) ChIP assays were performed with HDAC-1-VRPP-inducible cells transiently transfected with Flag-DPF2 and treated with or without Dox for $36 \mathrm{~h}$ using anti-Flag antibody as above. Data are represented as means of \% input \pm SD of technical replicates. $P \leq 0.05$. (F) ChIP assays were performed with HDAC-1-VRPP-inducible cells transiently transfected with BRD4 and treated with or without Dox for $36 \mathrm{~h}$ using anti-BRD4 antibody as above. Data are represented as means of $\%$ input \pm SD of technical replicates. $P \leq 0.05$. 
consistently affect the association of BRD4 with gene promoters (Figure 6F). Thus, selective histone decrotonylation results in reduced promoter association of crotonylation readers AF9 and DPF2 but not the acetylation reader BRD4.

Histone crotonylation is enriched in and required for self-renewal of ESCs

By WB analysis of core histones prepared from mouse ESCs and differentiated embryoid bodies (EB), we detected a much higher level of histone crotonylation in CGR8 ESCs (Figure 7A). Consistent with previous reports [31, 32], we also detected a much higher level of histone acetylation in ESCs (Figure 7A). This observation raised a question as to the role of enriched histone crotonylation in ESCs. To this end, we generated stable ESC lines expressing Dox-inducible HA-tagged HDAC1 or HDAC1-VRPP (Figure 7B). WB analysis using anti-HDAC1 antibody that detected both endogenous and HA-tagged HDAC1 revealed that Dox treatment for 36 $\mathrm{h}$ increased the levels of HDAC1 by 2-3-folds (Figure 7B). Notably, we observed that treatment with Dox resulted in marked differentiation of both HDAC1- and HDAC1-VRPP-expressing ESCs but not the control ESCs (Figure 7C). This Dox-induced differentiation was further confirmed by WB analysis of ESCs treated with Dox for various time periods. As shown in Figure 7D, induced expression of both HDAC1 and HDAC1VRPP resulted in a drastic reduction of Sox2, Oct4 and Nanog, the core transcription factors required for ESC pluripotency [33-35], thus supporting a differentiation phenotype observed in Figure 7C. WB analysis of core histones revealed that Dox-induced expression of both HDAC1 and HDAC-VRPP resulted in marked reduction of histone crotonylation (Figure 7D). As expected, Dox-induced WT HDAC1 expression also led to a marked reduction of histone acetylation. Interestingly, Dox-induced HDAC1-VRPP expression also resulted in reduced levels of histone acetylation, although less dramatic in comparison to induced WT HDAC1. Because HDAC1-VRPP lacks HDAC activity, we suggest that the observed reduction in histone acetylation is likely a consequence of ESC differentiation upon histone decrotonylation, since a reduced level of histone acetylation was reported for differentiated ESCs [31, 32].

To further substantiate the differentiation phenotype induced by histone decrotonylation, we examined the expression of marker genes for endoderm, mesoderm and ectoderm $[33,35]$. As shown in Figure 7E, qRT-PCR analysis revealed that Dox treatment induced progressively the expression of endoderm markers FOXA2 and AFP, mesoderm markers MIX1 and FOXH1, and ecto- derm markers PAX6 and OTX1. Thus, enriched histone crotonylation in ESCs is required for self-renewal of ESCs, as selective histone decrotonylation leads to ESC differentiation.

\section{Discussion}

Recent findings on various types of histone acylation have significantly expanded the repertoire of epigenetic modifications [9]. The identification of crotonylation-selective reader proteins further inspires the interest on this non-acetyl histone acylation $[11-13,36]$. In this study we demonstrate that class I HDACs are the major enzymes for histone decrotonylation in cells. Furthermore, we have provided compelling evidence that histone crotonylation is as dynamic as histone acetylation and is not functionally redundant to acetylation but critically important for global transcription in mammalian cells.

Through screening ectopically expressed HDAC and SIRT family proteins for HDCR activity in HeLa cells by IF staining using a pan-Kcr-specific antibody, we identified class I HDACs and SIRT1 as active HDCR enzymes (Figure 1). The HDCR activity for ectopically expressed class I HDACs and SIRT1 was substantiated by WB analysis using pan-Kcr-specific antibody (Figure $1 \mathrm{E})$, and by IF staining and WB analysis using histone Kcr site-specific antibodies (Figure 3). Notably, under the same experimental conditions, we failed to detect any significant HDCR activity for class II HDACs and class IV HDAC11 (Figure 1B-1C and Supplementary information, Figure S2A), indicating that HDCR activity is specific to class I but not class II and IV HDACs. In support of this, the HDCR activity for class I HDACs was demonstrated by loss-of-function RNA interference assay (Figure 2A) and by in vitro histone decrotonylation assay (Figure 4). Furthermore, as loss-of-function assay revealed that knockdown of class I HDACs, individually or in combination, resulted in clearly increased levels of histone crotonylation, class I HDACs are likely the major HDCR activity in mammalian cells (Figure 2A). This notion is further supported by the fact that treatment with TSA but not NAM markedly elevated the levels of histone crotonylation in various cells tested (Figure 2B-2D and Supplementary information, Figure S5). Thus, our study provides compelling evidence that class I HDACs are active histone decrotonylases and are most likely responsible for bulk histone decrotonylation in mammalian cells. The failure for previous studies to detect significant HDCR activity for the class I HDACs could be explained by in vitro-based assay used in their study [4]. Class I HDACs are well known for their function in the context of large multi-protein complexes [37-43] and purified re- 


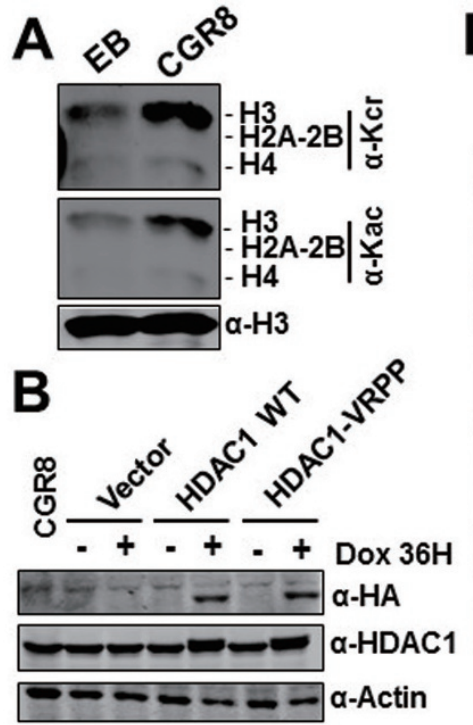

D $\frac{\text { HDAC1WT }}{3 D} \frac{\text { HDAC1-VRPP }}{3 D}$

$\overline{E+} \overline{-+}+\overline{-+} \overline{-+} \overline{-+} \overline{-+} \mathrm{Dox}$

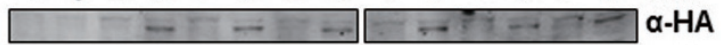

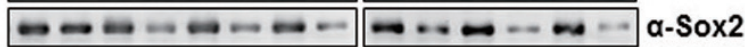

$\ldots \ldots \ldots+\cdots \cdots+\cdots$, act4

$--\cdots+\cdots+\cdots+\cdots+N a n o g$

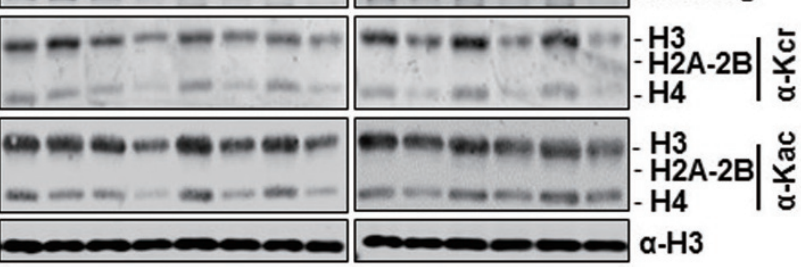

E
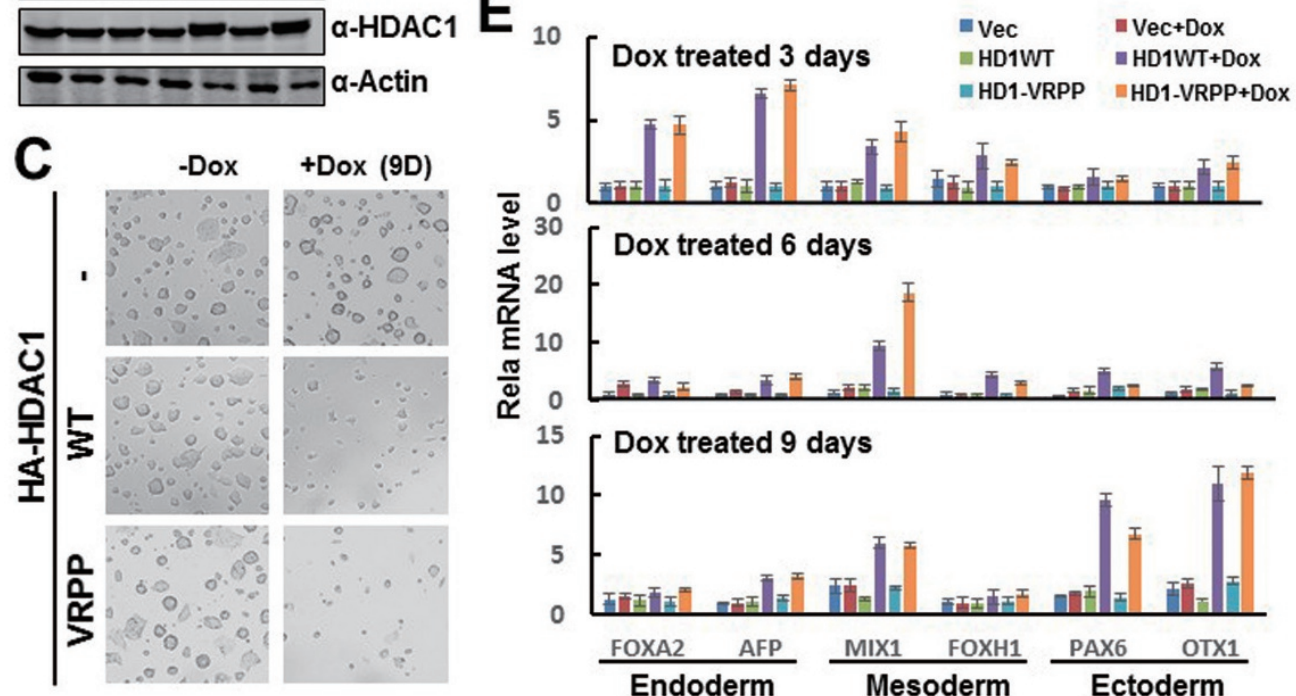

F
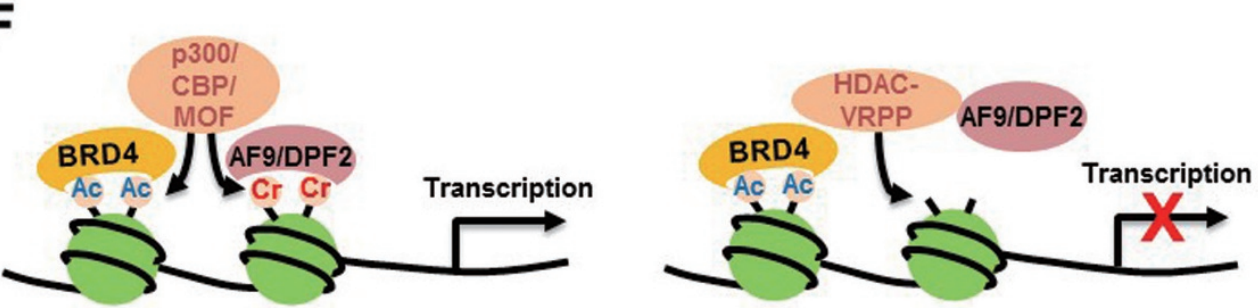

Figure 7 Histone crotonylation is enriched in and required for self-renewal of mESCs. (A) WB analysis showing a significantly higher level of histone crotonylation in mESC than in differentiated embryoid bodies (EB). CGR8 mESC were induced to differentiate by suspension culture in dish for 9 days. (B) WB analysis showing induced expression of WT HDAC1 and HDAC1-VRPP in CGR8 cells. HA antibody detected only Dox-induced HA-tagged HDAC1 or HDAC1-VRPP, whereas HDAC1 antibody detected both induced and endogenous HDAC1 proteins. (C) Contrast-phase images of control, WT HDAC1 and HDAC1-VRPP CGR8 colonies cultured for 9 days with or without Dox. (D) WB analysis showing the effect of induced expression of WT HDAC1 and HDAC1-VRPP on the levels of mESC core transcription factors Sox2, Oct4 and Nanog and histone crotonylation and histone acetylation. Note that reduced levels of Sox2, Oct4, Nanog, histone crotonylation and histone acetylation were observed upon 3 days of Dox treatment. (E) Confirmation of induced differentiation upon Dox-induced expression of WT HDAC1 or HDAC1-VRPP by qRT-PCR analysis of indicated differentiation marker genes. (F) Working model illustrating a non-redundant function of histone crotonylation to histone acetylation in transcription. CBP/p300 and MOF catalyze both histone acetylation and crotonylation, which in turn recruit corresponding reader proteins such as BRD4 or DPF2 and AF9, and facilitate transcriptional activation (left panel). Selective decrotonylation by HDCR1-VRPP is sufficient to repress transcription, indicating that histone crotonylation is required for transcriptional activation (right panel). 
combinant HDAC proteins usually display poor activity.

The detection of HDCR activity for SIRT1 in our study is in agreement with previous in vitro-based studies on HDCR activity of SIRT1 [23]. Although SIRT2 and SIRT3 were shown to decrotonylate histone peptides in vitro [23] and SIRT3 was reported to decrotonylate H3K$4 \mathrm{cr}$ in living cells [24], we failed to detect any significant HDCR activity for both SIRT2 and SIRT3. This discrepancy could be explained by potential difference in assay sensitivity. In vitro-based HDCR assays are likely more sensitive and thus more likely to detect weak HDCR activity. Nevertheless, both our ectopic overexpression (gain-of-function) and RNA interference (loss-of-function) assays failed to detect significant HDCR activity for SIRT2 and SIRT3 (Supplementary information, Figures S2B and S3), suggesting they have only weak, if any, HDCR activity.

Although SIRT1 and class I HDACs are active histone decrotonylases, our data indicate that they likely exhibit distinct site specificity in histone decrotonylation (Figure 3). For example, while HDAC1 is active in decrotonylation of H3K4, H3K9, H3K18, H3K23, H4K8 and $\mathrm{H} 4 \mathrm{~K} 12$, SIRT1 is inactive for decrotonylation of H3K23 as well as H4K12. In addition, HDAC2, HDAC3 and HDAC8 appear to be also inactive for decrotonylation of H3K23. Since these experiments were performed under the same conditions, the observed differences in site specificity are likely true. Nevertheless, this observation needs to be validated in the future by assays independent of site-specific histone crotonylation antibodies used in our study.

Our study also suggests that histone crotonylation is as dynamic as histone crotonylation. TSA treatment led to increases of histone crotonylation and acetylation in a similar fashion (Figure 2C-2D and Supplementary information, Figure S5), whereas treatment of cells with the $\mathrm{CBP} / \mathrm{p} 300$-selective inhibitor C646 led to decrease of histone crotonylation and acetylation in a similar kinetics [54]. Together these data support the notion that the class I HDACs are the major histone decrotonylases and that CBP and p300 are the major HCTs.

The most important finding in our study is the demonstration that histone crotonylation is critically important for global transcription in mammalian cells. This conclusion is based on our functional analysis of the HDAC1VRPP mutant that is active in HDCR but inactive in HDAC activity (Figure 4). Although previous studies [10] and ours with $\mathrm{CBP} / \mathrm{p} 300$ mutants with defective HAT but competent HCR activity [54] provide compelling evidence that $\mathrm{CBP} / \mathrm{p} 300$ can promote transcription through their HCT activity, it is not known from these studies if histone crotonylation acts independently of histone acetylation or is functionally redundant to histone acetylation. Our fortuitous finding that the HDAC1-VRPP mutant is inactive in HDAC but active in HDCR allowed us to test the effect of selective histone decrotonylation on transcription. Although why this mutant loses the HDAC but maintains the HDCR activity awaits structural study, a potential explanation is that HDAC1-VRPP mutation allows a more planar and rigid crotonyl group but not the more flexible acetyl group to reach the narrower enzymatic active site, as suggested by a recent structural study on p300 complexes with acyl-CoA variants [44]. Importantly, our results indicate that the HDAC1-VRPP mutant is capable of repressing transcription activated by various transcription factors (Supplementary information, Figure S9). Furthermore, by establishing inducible stable cell lines expressing either wild-type or the HDAC1VRPP mutant, we are able to demonstrate a global effect of selective histone decrotonylation on transcription (Figure 5). Our results indicate that the HDAC1-VRPP mutant is capable of repressing global transcription essentially as effective as the wild-type HDAC1 (Figure 5). Moreover, since more than half of the total genes detected by RNA-seq in our experiments are repressed by induced expression of the wild-type and HDAC1-VRPP mutant, it implies that decrotonylation without deacetylation is able to repress transcription as broadly as both decrotonylation and deacetylation. Therefore, our results provide first evidence that histone crotonylation is not redundant to histone acetylation but is broadly essential for transcription. In support of this conclusion, our ChIP analysis revealed a nice correlation between promoter histone decrotonylation and transcriptional repression by HDAC1-VRPP (Figure 6A-6C). Of note, one caveat for our study is that we do not know if the HDAC1-VRPP mutant is able to remove the other type(s) of histone acylation. Thus, in principle we could not exclude the possibility that the HDAC1-VRPP mutant might repress transcription through deacylation other than decrotonylation. However, our ChIP analysis of crotonylation reader proteins demonstrated that promoter histone decrotonylation correlates with diminished association of selective crotonylation reader proteins AF9 and DPF2 but not acetylation reader protein BRD4 (Figure 6D-6F). Thus, our study supports a working model that, although histone crotonylation and acetylation are catalyzed by the same enzymes $(\mathrm{CBP} / \mathrm{p} 300$ and $\mathrm{MOF})$ and removed by the same enzymes (SIRT1 and class I HDACs), histone crotonylation plays a non-redundant role to histone acetylation, most likely through crotonylation-selective readers such as AF9 and DPF2 (Figure 7F). Future work should be devoted to elucidate whether and how histone crotonylation readers mediate the essential function of 
histone crotonylation in transcription.

Our RNA-seq analysis also reveals the existence of genes differentially influenced by wild-type HDAC1 and the HDAC1-VRPP1 mutant (Figure 5F-5I). These results imply that while the majority of genes are regulated by both acetylation and crotonylation, some genes could be preferentially regulated by acetylation or by crotonylation. However, our ChIP analysis revealed that promoter histone crotonylation was clearly less reduced by HDAC1-VRPP for genes downregulated by WT HDAC1 only. Thus, an alternative explanation is that this group of genes could be regulated by histone crotonylation as the other two groups. They were not downregulated by HDAC1-VRPP simply because their promoter histone crotonylation was not significantly affected by HDAC1VRPP. Thus, while our study has provided compelling evidence for a broad function of histone crotonylation in transcription, it remains to be determined if there are genes whose transcription is independent of histone crotonylation.

We also show that ESCs are enriched in histone crotonylation and that enriched histone crotonylation is required for maintenance of ESC self-renewal (Figure 7). Previously studies have shown that ESCs maintain elevated levels of histone acetylation by producing more acetyl-CoA through glycolysis [32]. Inhibition of glycolysis results in differentiation of ESCs. In addition, multiple HATs including p300 and MOF are required for open chromatin structure that correlates directly with pluripotency [34, 45-47]. Our finding that ESCs display an elevated level of histone crotonylation suggests the existence of metabolic pathway in ESCs that fuels crotonyl-CoA. Given our observation that selective histone decrotonylation caused differentiation of ESCs, we suggest that histone crotonylation also contributes to the formation and maintenance of ESC open chromatin. In this regard, the bulkier crotonyl residue is likely to have a bigger effect on chromatin structure than the acetyl residue. Differentiation induced by selective histone decrotonylation is also consistent with our observation that histone crotonylation has a critical and broad role in transcription.

In sum, our finding that histone crotonylation is not redundant to acetylation but is broadly essential for transcription demonstrates for the first time a type of lysine acylation other than acetylation could be essential for global transcriptional regulation in mammalian cells. Our finding that histone crotonylation is generally required for transcription also supports potential roles of histone crotonylation in other chromatin-associated events. The HDAC activity-deficient HDAC1/3-VRPP mutants identified in this study would be valuable tools for elucidating the molecular mechanisms and physiological functions of histone crotonylation.

\section{Materials and Methods}

\section{Cell lines, antibodies, reagents and plasmids}

HeLa, 293T and NIH3T3 cells were cultured in DMEM (GIBCO) with 10\% fetal bovine serum (FBS) (Intergen). A549 cells were cultured in 1640 (GIBCO) with $10 \%$ FBS. HCT116 cells were cultured in McCoy's 5A (GIBCO) with $10 \%$ FBS. ESCs (CGR8) were cultured on $0.1 \%$ gelatin-coated plates in GMEM ESC medium (GIBCO) containing 15\% FBS (GIBCO), $2 \mathrm{mM}$ L-glutamine (Hyclone), $100 \mathrm{mM}$ nonessential amino acids (Hyclone), $0.1 \mathrm{mM}$ 2-mercaptoethanol (GIBCO), $1 \mathrm{mM}$ sodium pyruvate and leukemia inhibitory factor (LIF, $1000 \mathrm{U} / \mathrm{ml}$, Chemicon). The following antibodies were used in this study: panKac (PTM-Biolabs 101), pan-Kcr (PTM-Biolabs 501), H3K4cr (PTM-Biolabs PTM-527), H3K9cr (PTM-Biolabs 516), H3K18cr (PTM-Biolabs 517), H3K23cr (PTM-Biolabs 519), H4K8cr (PTM-Biolabs 522), H4K12cr (PTM-Biolabs 523), H4ac (Millipore 05-1355), H3 (Epitomics M1309-1), Flag (Sigma 7425/1804), HA (Santa Cruz SC-805), Gal4DBD (Santa Cruz SC-510), GAPDH (Abmart M20006), actin (Huabio M1210-2), HDAC1 (ABclonal A0238), HDAC2 (ABclonal A2084) and HDAC3 (ABclonal A2139), BRD4 (Abcam ab128874), Sox2 (Abcam ab92494), Oct4 (Santa Cruz sc-5279) and Nanog (Abclonal A3232). TSA was from Selleck, NAM was from Beyotime, sodium butyrate was from Sigma. The plasmids of HA-HDAC1 and HA-HDAC3 were gifts from Dr Qunying Lei Laboratory. All mutants were generated by PCR-based point mutagenesis strategy and verified by DNA sequencing.

\section{Transfections, IF staining and WB analysis}

DNA and siRNA transient transfection were performed using lipofectamine 2000 (Invitrogen) according to the manufacturer's instructions. Histones were purified from cells using a standard acid extraction protocol [48]. IF staining and WB for various proteins was carried out essentially as described [49]. In brief, for IF staining HeLa cells on coverslips were washed with $1 \times$ PBS (137 $\mathrm{mM} \mathrm{NaCl}, 2.7 \mathrm{mM} \mathrm{KCl}, 10 \mathrm{mM} \mathrm{Na} \mathrm{HPO}_{4}$ and $\left.2 \mathrm{mM} \mathrm{KH}_{2} \mathrm{PO}_{4}\right)$ prior to fixation in $4 \%$ paraformaldehyde at room temperature for 30 min. The coverslips were incubated with $1 \%$ Triton X-100 on ice for $15 \mathrm{~min}$, blocked with $5 \% \mathrm{BSA}$ in $37{ }^{\circ} \mathrm{C}$ incubator for 60 min and incubated with mouse or rabbit anti-Flag/HA antibody for $2 \mathrm{~h}$. The coverslips were washed three times with PBST, followed by incubation with Texas green-conjugated secondary antibody against mouse or rabbit. Images were acquired with an Olympus microscope system.

\section{Immunoaffinity purification}

For immunoaffinity purification WT and mutant HDAC1, 293T cells transfected with expression plasmids of interest were washed with ice-cold PBS and lysed with $0.25 \mathrm{ml}$ ice-cold lysis buffer (50 mM Tris-HCl, pH 8.0, 1\% Triton X-100; 150 mM NaCl, 5 mM EDTA, protease inhibitor cocktail) by incubating on ice for 5 min. The lysates were scraped off and transferred to microcentrifuge tubes, and further lysed on ice for $30 \mathrm{~min}$. The lysates were centrifuged for $10 \mathrm{~min}$ at $14000 \times \mathrm{g}$ at $4{ }^{\circ} \mathrm{C}$, and the supernatant was transferred to a new tube. For each $150 \mu$ l of cell lysate, $8 \mu 1$ 
M2, MYC or HA antibody-conjugated beads were added and equilibrated with $200 \mu \mathrm{l}$ binding buffer (50 mM Tris- $\mathrm{HCl}, \mathrm{pH} 7.5,0.1 \%$ Triton X-100, $150 \mathrm{mM} \mathrm{NaCl}, 5 \mathrm{mM}$ EDTA, 10\% glycerol, protease inhibitor cocktail). The tubes were gently rocked overnight at $4{ }^{\circ} \mathrm{C}$. The beads were washed three times with $500 \mu$ of washing buffer (50 mM Tris-HCl, pH 7.5, 0.1\% Triton X-100, $150 \mathrm{mM} \mathrm{NaCl}, 5 \mathrm{mM}$ EDTA and protease inhibitor cocktail) and used for in vitro enzymatic assays.

\section{In vitro $H D A C$ and $H D C R$ assays}

For in vitro deacetylation and decrotonylation assays, HA-tagged WT or mutant HDAC1 proteins were expressed and purified from $293 \mathrm{~T}$ cells. HDAC and HDCR assays were carried out at $37{ }^{\circ} \mathrm{C}$ for $12 \mathrm{~h}$ in reaction buffer $(50 \mathrm{mM}$ Tris $\mathrm{pH} 8.0,137 \mathrm{mM} \mathrm{NaCl}, 2.7$ $\mathrm{mM} \mathrm{KCl}, 1 \mathrm{mM} \mathrm{MgCl}$ and $1 \mathrm{mM}$ DTT). Crotonylated and acetylated core histones were prepared from sodium crotonate (Sigma MP207938) and TSA + NAM-treated HeLa cells. The products were then subjected to WB analysis with anti-pan-Kac and anti-pan-Kcr antibody.

Quantitative RT-PCR, siRNAs and luciferase reporter assay

For qRT-PCR analysis, total RNAs were prepared by RNA preparation kit (TransGen). The primers for qRT-PCR are listed in Supplementary information, Table S1. The siRNAs targeting human HDAC1, HDAC2, HDAC3, SIRT1, SIRT3 and SIRT5 used in this study were provided in Supplementary information, Table S3; all siRNAs were ordered from GenePharma. Luciferase reporter assay was performed using a dual luciferase reporter assay kit from Promega.

\section{Chromatin immunoprecipitation assay}

Cross-linking ChIP was performed with anti-H3K18cr, anti-H4ac, anti-Flag and anti-BRD4 essentially as described [50].

\section{RNA-seq analysis}

RNA-seq analysis was performed by Berry Genomics (Beijing, China). All the RNA-Seq data sets were mapped onto yeast genome (sacCer3) for control spike-in RNAs and human genome (hg19) using tophat2 (version 2.1.0) respectively. Genome sequence files (hg19.2bit, sacCer3.2bit) and annotation files were downloaded from UCSC. The HTSeq (version 0.6.0) was used to quantify mapped counts of yeast and human genes separately. For normalizing human gene expression, we estimated size factors of every RNA-Seq data set with yeast gene counts using $\mathrm{R}$ package DESeq2, and then adjusted human gene counts using corresponding size factors.

\section{EB formation differentiation assay}

ESCs were harvested by trypsinization, and aliquots of $3 \times 10^{5}$ cells were resuspended in 6-cm special culture dishes in ESC medium without LIF to generate EBs. The medium was changed every day, and a portion of the cells was discarded to maintain the proper density. The generated EBs were seeded onto 10-cm dishes for differentiation. Histones were purified from the EBs at the indicated days of differentiation using a standard acid extraction and used for WB analysis.

\section{Acknowledgments}

We thank members of Wong's laboratory for valuable discussion.
We thank Dr Cheng-Ming Chiang (UT Southwestern) for critical reading of the manuscript. This study is supported by grants from the National Science and Technology Major Project "Key New Drug Creation and Manufacturing Program" of China (2014ZX09507002005 to JW), the Ministry of Science and Technology of China (2015CB910402 to JW), the National Natural Science Foundation of China (81530078 and 31571325 to JW), the Science and Technology Commission of Shanghai Municipality (14XD1401700 and 11DZ2260300 to JW).

\section{Author Contributions}

Wei Wei and Xiaoguang Liu designed and performed most of the experiments, analyzed data and wrote the manuscript. Jiwei Chen and Tieliu Shi performed all RNA-seq data analysis. Lu Lu, Huifang Zhang and Guangjin Ding participated in screening for HDCRs. Shennan Gao and Zhiqiang Wang performed ES cells-related experiments. Zhongzhou Chen designed HDAC1 mutant. Jiwen Li, Jianjun $\mathrm{Yu}$ and Jiemin Wong supervised the study, and Jiemin Wong wrote the manuscript together with Wei Wei and Xiaoguang Liu.

\section{Competing Financial Interests}

The authors declare no competing financial interests.

\section{References}

1 Roth SY, Denu JM, Allis CD. Histone acetyltransferases. Annu Rev Biochem 2001; 70:81-120.

2 Wolffe AP. Histone deacetylase: a regulator of transcription. Science 1996; 272:371-372.

3 Rousseaux S, Khochbin S. Histone acylation beyond acetylation: terra incognita in chromatin biology. Cell J 2015; 17:1-6.

4 Tan M, Luo H, Lee S, et al. Identification of 67 histone marks and histone lysine crotonylation as a new type of histone modification. Cell 2011; 146:1016-1028.

5 Olsen CA. Expansion of the lysine acylation landscape. Angew Chem Int Ed Engl 2012; 51:3755-3756.

6 Dai L, Peng C, Montellier E, et al. Lysine 2-hydroxyisobutyrylation is a widely distributed active histone mark. Nat Chem Biol 2014; 10:365-370.

7 Zhang K, Chen Y, Zhang Z, Zhao Y. Identification and verification of lysine propionylation and butyrylation in yeast core histones using PTMap software. J Proteome Res 2009; 8:900906.

8 Xie Z, Dai J, Dai L, et al. Lysine succinylation and lysine malonylation in histones. Mol Cell Proteomics 2012; 11:100-107.

9 Sabari BR, Zhang D, Allis CD, Zhao Y. Metabolic regulation of gene expression through histone acylations. Nat Rev Mol Cell Biol 2017; 18:90-101.

10 Sabari BR, Tang Z, Huang H, et al. Intracellular crotonyl-CoA stimulates transcription through $\mathrm{p} 300$-catalyzed histone crotonylation. Mol Cell 2015; 58:203-215.

11 Li Y, Sabari BR, Panchenko T, et al. Molecular coupling of histone crotonylation and active transcription by AF9 YEATS domain. Mol Cell 2016; 62:181-193.

12 Xiong X, Panchenko T, Yang S, et al. Selective recognition of histone crotonylation by double PHD fingers of MOZ and DPF2. Nat Chem Biol 2016; 12:1111-1118.

13 Andrews FH, Shinsky SA, Shanle EK, et al. The Taf14 YEATS domain is a reader of histone crotonylation. Nat Chem Biol 
2016; 12:396-398.

14 Li Y, Wen H, Xi Y, et al. AF9 YEATS domain links histone acetylation to DOT1L-mediated H3K79 methylation. Cell 2014; 159:558-571.

15 Zeng L, Zhang Q, Li S, Plotnikov AN, Walsh MJ, Zhou MM. Mechanism and regulation of acetylated histone binding by the tandem PHD finger of DPF3b. Nature 2010; 466:258-262.

16 Zhao D, Guan $\mathrm{H}$, Zhao $\mathrm{S}$, et al. YEATS2 is a selective histone crotonylation reader. Cell Res 2016; 26:629-632.

17 Cheng Z, Tang Y, Chen Y, et al. Molecular characterization of propionyllysines in non-histone proteins. Mol Cell Proteomics $2009 ; 8: 45-52$.

18 Smith BC, Denu JM. Acetyl-lysine analog peptides as mechanistic probes of protein deacetylases. J Biol Chem 2007; 282:37256-37265.

19 Du J, Zhou Y, Su X, et al. Sirt5 is a NAD-dependent protein lysine demalonylase and desuccinylase. Science 2011; 334:806809.

20 Park J, Chen Y, Tishkoff DX, et al. SIRT5-mediated lysine desuccinylation impacts diverse metabolic pathways. Mol Cell 2013; 50:919-930.

21 Rardin MJ, He W, Nishida Y, et al. SIRT5 regulates the mitochondrial lysine succinylome and metabolic networks. Cell Metab 2013; 18:920-933.

22 Tan M, Peng C, Anderson KA, et al. Lysine glutarylation is a protein posttranslational modification regulated by SIRT5. Cell Metab 2014; 19:605-617.

23 Feldman JL, Baeza J, Denu JM. Activation of the protein deacetylase SIRT6 by long-chain fatty acids and widespread deacylation by mammalian sirtuins. J Biol Chem 2013; 288:31350-31356.

24 Bao X, Wang Y, Li X, et al. Identification of 'erasers' for lysine crotonylated histone marks using a chemical proteomics approach. eLife 2014; 3. doi: 10.7554/eLife.02999

25 Madsen AS, Olsen CA. Profiling of substrates for zinc-dependent lysine deacylase enzymes: HDAC3 exhibits decrotonylase activity in vitro. Angew Chem Int Ed Engl 2012; 51:9083-9087.

26 Van der Veer E, Ho C, O’Neil C, et al. Extension of human cell lifespan by nicotinamide phosphoribosyltransferase. J Biol Chem 2007; 282:10841-10845.

27 Lin CY, Loven J, Rahl PB, et al. Transcriptional amplification in tumor cells with elevated c-Myc. Cell 2012; 151:56-67.

28 Loven J, Orlando DA, Sigova AA, et al. Revisiting global gene expression analysis. Cell 2012; 151:476-482.

29 Dey A, Chitsaz F, Abbasi A, Misteli T, Ozato K. The double bromodomain protein Brd4 binds to acetylated chromatin during interphase and mitosis. Proc Natl Acad Sci USA 2003; 100:8758-8763.

30 Filippakopoulos P, Picaud S, Mangos M, et al. Histone recognition and large-scale structural analysis of the human bromodomain family. Cell 2012; 149:214-231.

31 Kimura H, Tada M, Nakatsuji N, Tada T. Histone code modifications on pluripotential nuclei of reprogrammed somatic cells. Mol Cell Biol 2004; 24:5710-5720.

32 Moussaieff A, Rouleau M, Kitsberg D, et al. Glycolysis-mediated changes in acetyl-CoA and histone acetylation control the early differentiation of embryonic stem cells. Cell Metab 2015; 21:392-402.

33 Niwa H, Miyazaki J, Smith AG. Quantitative expression of Oct-
3/4 defines differentiation, dedifferentiation or self-renewal of ES cells. Nat Genet 2000; 24:372-376.

34 Young RA. Control of the embryonic stem cell state. Cell 2011; 144:940-954.

35 Fang L, Zhang L, Wei W, et al. A methylation-phosphorylation switch determines Sox2 stability and function in ESC maintenance or differentiation. Mol Cell 2014; 55:537-551.

36 Li Y, Zhao D, Chen Z, Li H. YEATS domain: linking histone crotonylation to gene regulation. Transcription 2017; 8:9-14.

37 Zhang Y, Iratni R, Erdjument-Bromage H, Tempst P, Reinberg D. Histone deacetylases and SAP18, a novel polypeptide, are components of a human Sin3 complex. Cell 1997; 89:357-364.

38 Hassig CA, Fleischer TC, Billin AN, Schreiber SL, Ayer DE. Histone deacetylase activity is required for full transcriptional repression by mSin3A. Cell 1997; 89:341-347.

39 Laherty CD, Yang WM, Sun JM, Davie JR, Seto E, Eisenman $\mathrm{RN}$. Histone deacetylases associated with the mSin 3 corepressor mediate mad transcriptional repression. Cell 1997; 89:349356.

40 Xue Y, Wong J, Moreno GT, Young MK, Cote J, Wang W. NURD, a novel complex with both ATP-dependent chromatin-remodeling and histone deacetylase activities. Mol Cell 1998; 2:851-861.

41 Zhang Y, LeRoy G, Seelig HP, Lane WS, Reinberg D. The dermatomyositis-specific autoantigen $\mathrm{Mi} 2$ is a component of a complex containing histone deacetylase and nucleosome remodeling activities. Cell 1998; 95:279-289.

42 Guenther MG, Lane WS, Fischle W, Verdin E, Lazar MA, Shiekhattar R. A core SMRT corepressor complex containing HDAC3 and TBL1, a WD40-repeat protein linked to deafness. Genes Dev 2000; 14:1048-1057.

43 Li J, Wang J, Wang J, et al. Both corepressor proteins SMRT and $\mathrm{N}-\mathrm{CoR}$ exist in large protein complexes containing HDAC3. EMBO J 2000; 19:4342-4350.

44 Kaczmarska Z, Ortega E, Goudarzi A, et al. Structure of p300 in complex with acyl-CoA variants. Nat Chem Biol 2017; 13:21-29.

45 Yao TP, Oh SP, Fuchs M, et al. Gene dosage-dependent embryonic development and proliferation defects in mice lacking the transcriptional integrator p300. Cell 1998; 93:361-372.

46 Chelmicki T, Dundar F, Turley MJ, et al. MOF-associated complexes ensure stem cell identity and Xist repression. eLife 2014; 3:e02024.

47 Li X, Li L, Pandey R, et al. The histone acetyltransferase MOF is a key regulator of the embryonic stem cell core transcriptional network. Cell Stem Cell 2012; 11:163-178.

48 Shechter D, Dormann HL, Allis CD, Hake SB. Extraction, purification and analysis of histones. Nat Protoc 2007; 2:1445-1457.

49 Liu X, Gao Q, Li P, et al. UHRF1 targets DNMT1 for DNA methylation through cooperative binding of hemi-methylated DNA and methylated H3K9. Nat Commun 2013; 4:1563.

50 Yoon HG, Choi Y, Cole PA, Wong J. Reading and function of a histone code involved in targeting corepressor complexes for repression. Mol Cell Biol 2005; 25:324-335.

51 Millard CJ, Watson PJ, Celardo I, et al. Class I HDACs share a common mechanism of regulation by inositol phosphates. Mol Cell 2013; 51:57-67.

52 Schuetz A, Min J, Allali-Hassani A, et al. Human HDAC7 harbors a class IIa histone deacetylase-specific zinc binding motif 
and cryptic deacetylase activity. J Biol Chem 2008; 283:1135511363.

53 Vannini A, Volpari C, Gallinari P, et al. Substrate binding to histone deacetylases as shown by the crystal structure of the HDAC8-substrate complex. EMBO Rep 2007; 8:879-884.

54 Liu XG, Wei W, Liu YT, et al. MOF as an evolutionarily conserved histone crotonyltransferase and transcriptional activation by histone acetyltransferase-deficient and crotonyltransferase-competent CBP/p300. Cell Discov 2017; 3:17016. doi:10.1038/celldisc.2017.16

(Supplementary information is linked to the online version of the paper on the Cell Research website.) 